\title{
Algorithm of numerical optimization of steel structures on basis of minimum weight criterion
}

\author{
Tatiana Dmitrieva ${ }^{1, *}$ \\ ${ }^{1}$ Irkutsk National Research Technical University, 664074, Lermontova str., 83, Irkutsk, Russia
}

\begin{abstract}
The description of the algorithm of steel structures optimal design is described in the article. The calculation model is represented by flat and spatial rod elements. The problem of optimization is resolved with the help of mathematical programming. The material consumption is taken as a criterion of optimality. A wide range of inspections is presented in the form of regulatory requirements for strength, rigidity and local stability. The geometry parameters of the sections are varied, as well as the external geometry of the nodes of the final element model. Both continuous and discrete variations might be possible. The algorithm is implemented in two versions: on the basis of imitating the short-term problem and with the direct reference to the target and restrictive functions at each step of optimization process. The results of the solution of the steel framework optimization problem during on-going and discrete variations of geometric parameters are presented. The solutions obtained are estimated on uniqueness.
\end{abstract}

\section{Introduction}

One of the most intensively developing directions in the field of optimal design for the last 50 years has been the development of algorithms of building structures optimization [1-3]. The complex of tasks in this direction can be divided into several categories:

- the structures optimization by the type of material where traditionally the greatest attention is paid to metal structures optimization [4-8];

- by a set of techniques used in problems optimization;

- by the choice of the optimization criterion.

Meanwhile, it should be noted that in the design of building objects optimization algorithms do not find real implementation. In this paper we consider the option of embedding the optimization block in the algorithm for the automated calculation of planar and spatial rod systems.

\section{Relevance of the problem}

The optimization calculation involves the selection of parameters of a structure (or a building as a whole) according to the accepted criterion. The most objective criterion of

\footnotetext{
*Corresponding author: dmitrievat@ list.ru
} 
"optimality" undoubtedly is the cost. However, the formalization of such a criterion which determines the full cost of a building erecting structure from "zero", taking into account technological, transportation and other costs, is rather difficult.

That is why, a narrower problem of estimating the design in terms of the volume of the material is set. Such a criterion is quite in demand in construction practice because it directly affects the design of foundations. In most cases it reduces the cargo transportation costs, reduces the costs of erection technology etc. It is believed that the solution of this problem according to this criterion has been reached if the optimal design complies with all the regulatory requirements for the behavior of the structure, such as the requirements for strength, rigidity, local and general stability.

An important problem associated with the practical application of optimization techniques is the fact that optimization algorithms are not built into modern software complexes for automated building structures design. The most actively used in the Russian PC design constructions (LIRA SAPR, CSAD, Ing +, etc.) solve the problem of engineering analysis, i.e. allow us to determine the tense-deformed condition (TDC) of structures with known geometry. The algorithm presented in this article aims to select geometric parameters of the structure in accordance with a given criterion of optimality, taking into account the requirements defined by the design standards.

The lack of optimization blocks in software complexes is associated with the laboriousness of their implementation. The algorithms for searching the optimal projects require a multiple solution of the problem of finite element analysis of the structure of design model [9-11], and consequently they are rather cumbersome for systems with a large number of nodes (where, for example, plate-type constructions can be attributed to). The amount of computational operations is also determined by the number of parameters variation which include the geometry of the sections and certain nodal points of the design scheme. Varying the cross-section of each structural element leads to the fact that the number of these parameters can be measured by hundreds of thousands. However, a limited number of standard sizes (even for complex structures) reduce this task to reasonable limits. As a rule, only those parameters that have a significant effect on the TDC of the structure vary. The dimension of the optimization problem is also affected by the number of regulatory requirements for the operation structures. The list of requirements is formed for each group of standard sizes.

\section{Optimization problem setting}

There are many optimization algorithms sets. One of the most well-known approaches suggests the setting in the form of a problem of mathematical programming (MP) [12-14] find find use (1) if with restrictions (2):

$$
\begin{aligned}
& \min f(x, P(x)), \quad x \in E n \\
& g_{j}(x, P(x)) \leq 0, \quad j=1,2, \ldots, m ; \\
& x_{i}^{L} \leq x_{i} \leq x_{i}^{U}, \quad i=1,2, \ldots, n .
\end{aligned}
$$

The objective function $f$ represents the volume of the constructions. Vector $\{X\}-$ is a set of variation parameters, the range of which is specified by the vectors $\left\{X^{L}\right\},\left\{X^{U}\right\}$.

The problem of implementing the algorithm is that the constraint functions are related to the geometric parameters of the variation through the parameters of the state $P(\mathrm{x})$. These 
parameters can represent displacement functions $(\delta)$, internal forces $(M, Q, N)$, stresses $(\sigma)$, natural frequencies $(\omega)$, etc.

$$
\{P(x)\}=\varphi(\delta, M, Q, N, \sigma, \omega),
$$

To specify this problem it is necessary to solve the statistic analysis task taken in this algorithm as line setting:

$$
[K]\{\delta\}=\{F\} .
$$

\section{Solving task algorithms}

The general scheme of the algorithm for optimizing designs in static setting in 2 variants is presented here.

In the first variant in order to obtain explicit dependences of the functions (1-2) from the variable parameters an approximate problem is formed by constructing approximations of the target and limiting functions or the state parameters included into these functions. Fig. 1 depicts the relationship of the main blocks for this variant.

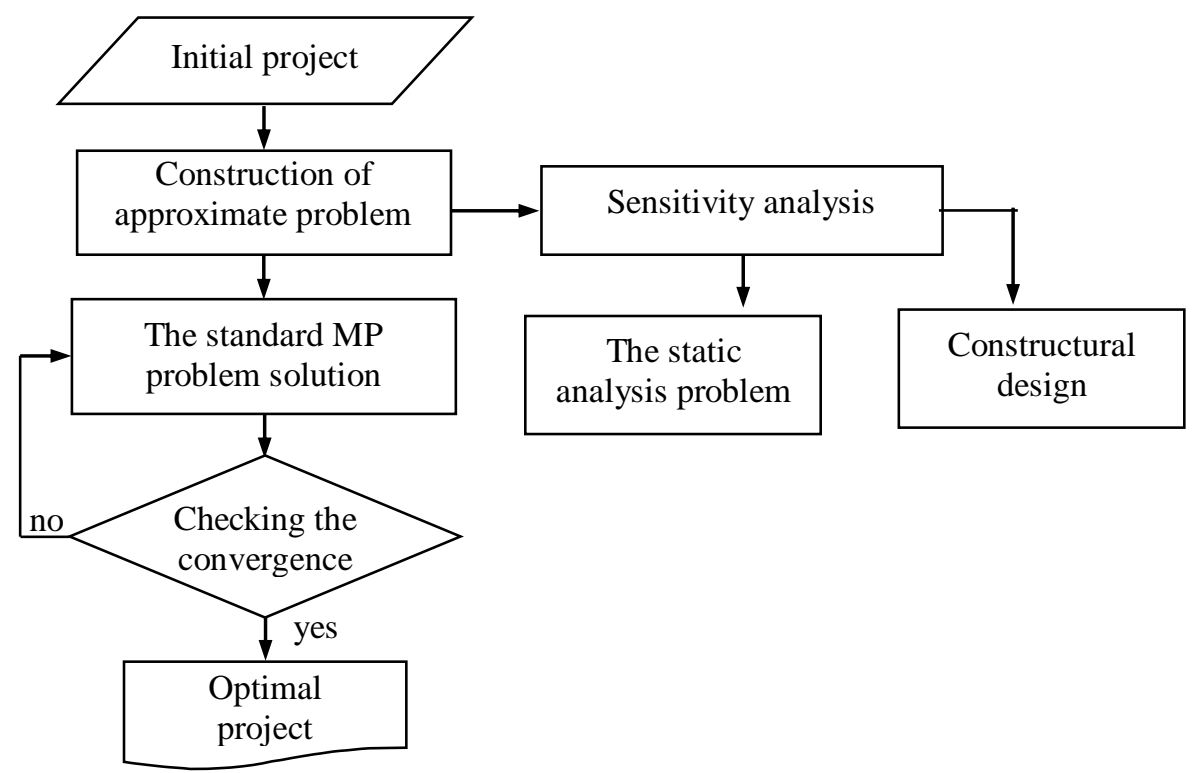

Fig. 1. Optimization algorithm on the approximations basis.

The approximation of the first order is performed by expansion in a Taylor series in a neighborhood of the point $x^{*}$ :

$$
\tilde{P}_{k}(x)=P_{k}\left(x^{*}\right)+\left\{\begin{array}{c}
\partial P_{k} \\
\partial x
\end{array}\right\}_{x=x *}^{T}\left\{x-x^{*}\right\}
$$

Such an approximation gives a good convergence to the optimum in case of variation of geometric characteristics of the cross sections provided that the strength constraints are 
given. If the external geometry of the structure varies with constraints on the rigidity, then the optimization problem becomes essentially non-linear. The best convergence in this case is given by the second-order approximations:

$$
\tilde{P}_{k}(x)=P_{k}\left(x^{*}\right)+\left\{\frac{\partial P_{k}}{\partial x}\right\}_{x=x^{*}}^{T}\left\{x-x^{*}\right\}+\frac{1}{2}\left\{x-x^{*}\right\}^{T}\left\{\frac{\partial^{2} P_{k}}{\partial x^{2}}\right\}_{x=x^{*}}^{T}\left\{x-x^{*}\right\} .
$$

The approximation coefficients are calculated on the basis of the sensitivity analysis methods. The second variant of the algorithm assumes that the optimization block appeals to the calculation of the target and limiting functions directly at each step of the solution of the standard MP problem (Fig. 2).

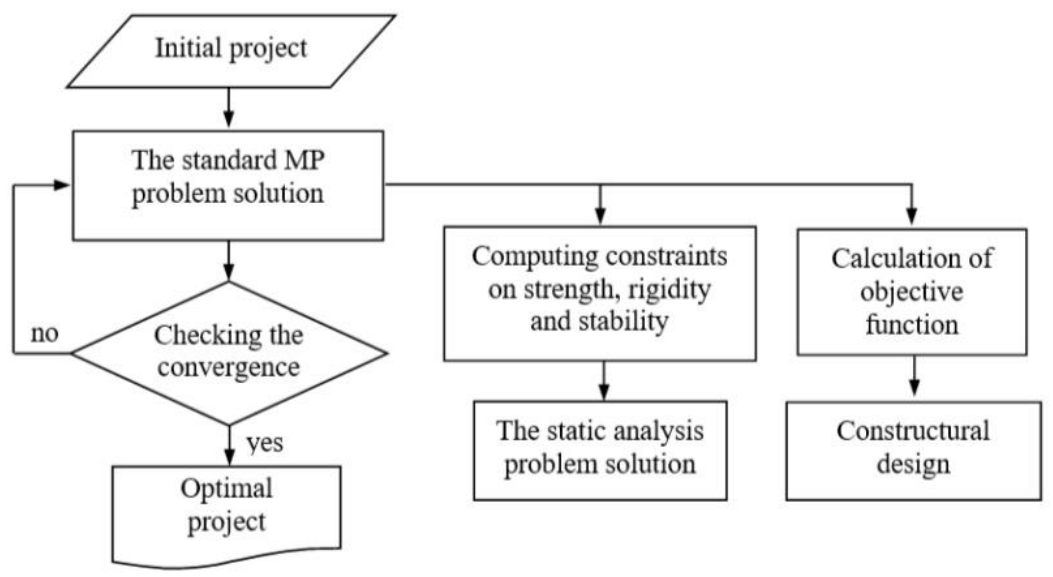

Fig. 2. Optimization algorithm for direct calculation of the goal function and constraints.

This approach can be implemented in solving problems of limited dimension, for example, in optimal design of rod systems, when the power of computing means allows finding the values of forces and displacements by finite element analysis for each calculation of the constraint functions. The algorithms implemented in the MP block are based on the methods of modified Lagrange functions [15].

\section{Parameters being varied in the optimization process}

As it has been already noted the parameters of the cross sections of the elements are selected as well as the coordinates of the nodes of the calculation scheme. There might be continuous and discrete changes. Any parameter can be fixed if the upper and lower bounds are set equal.

Let us dwell on options for variation of the parameters of the cross-sections of steel structures elements. Among them we can distinguish the base sections as well as the complex ones composed of the base sections (Table 1).

Here the variant of work with discrete parameters of cross-sections from range of metal structures is provided. For this purpose special databases are built into the algorithm which includes not the complete assortment of sections, but their selective values which ensure a monotonic increase in the areas and moments of inertia of the sections. 
Table 1. The schemes of section.

\begin{tabular}{|l|l|l|l|}
\hline & Simple (basic) sections & & \multicolumn{1}{|c|}{ Complex sections } \\
\hline $\mathbf{T}$ & Composite or rolled I-beam & $\mathbf{I}$ & 2 I-beam \\
\hline $\mathbf{Z}$ & Composite or rolling channel & & 2 channel \\
\hline$\square$ & Ring & $\mathbf{C I}$ & Channel + I-beam \\
\hline$\square$ & Equilateral box & $\mathbf{J}$ & 2 Corner \\
\hline$\square$ & The uneven box & 4 Corner \\
\hline & Corner & & \\
\hline
\end{tabular}

Fig. 3 shows the variants of variation of the calculation scheme by the coordinates of the nodes as well as the angle of inclination of the node line (Fig. 3).
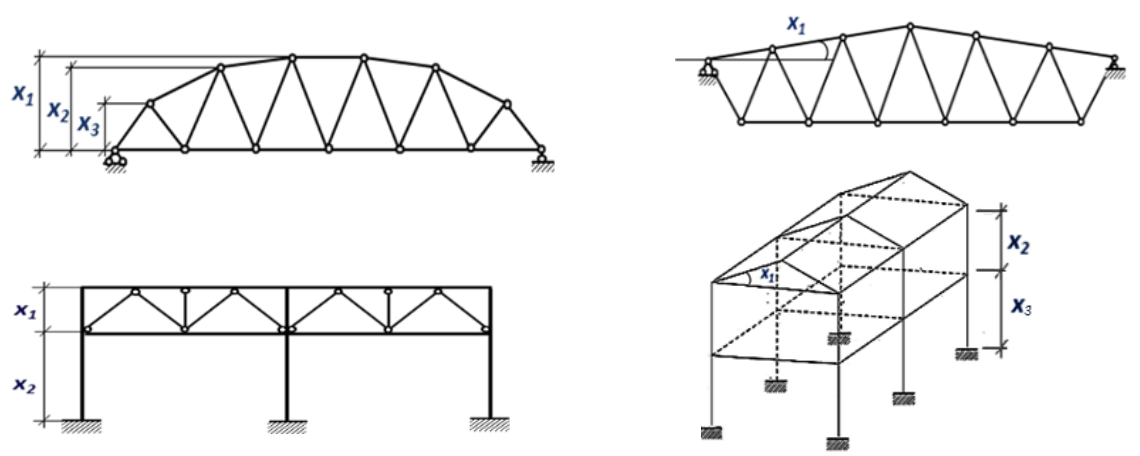

Fig. 3. Possible variants of variable parameters of external geometry.

\section{List of constraints in steel structures optimization}

The constraint functions $\mathrm{g}_{\mathrm{j}}(\mathrm{x})$ are requirements for strength and local stability in accordance with the normative documents for the calculation of steel structures. Here is a list of software modules that implement these requirements. Each module corresponds to a specific case of tense-deformed condition (TDC) reflected in Table 3.

Table 3. Types of tense-deformed condition (TDC).

\begin{tabular}{|c|l|l|}
\hline № & \multicolumn{1}{|c|}{ Type TDC } & Name module \\
\hline 1 & Central stretching-compression & Press_tens \\
\hline 2 & Bending in one plane & Bend_1p \\
\hline 3 & Bending in two planes & Bend_2p \\
\hline 4 & $\begin{array}{l}\text { Bending in one plane taking into account plastic } \\
\text { deformations }\end{array}$ & Bend_plast_lp \\
\hline 5 & $\begin{array}{l}\text { Bending in two planes taking into account plastic } \\
\text { deformations }\end{array}$ & Bend_plast_2p \\
\hline 6 & Compression with bending & Press_bend \\
\hline
\end{tabular}

The type of the stress-strain state is given by the variable TDC. A block diagram of the constraint generation algorithm for one group of elements $\left\{g_{-} e l\left(m_{-} e l\right)\right\}$ is shown in Fig. 4. 


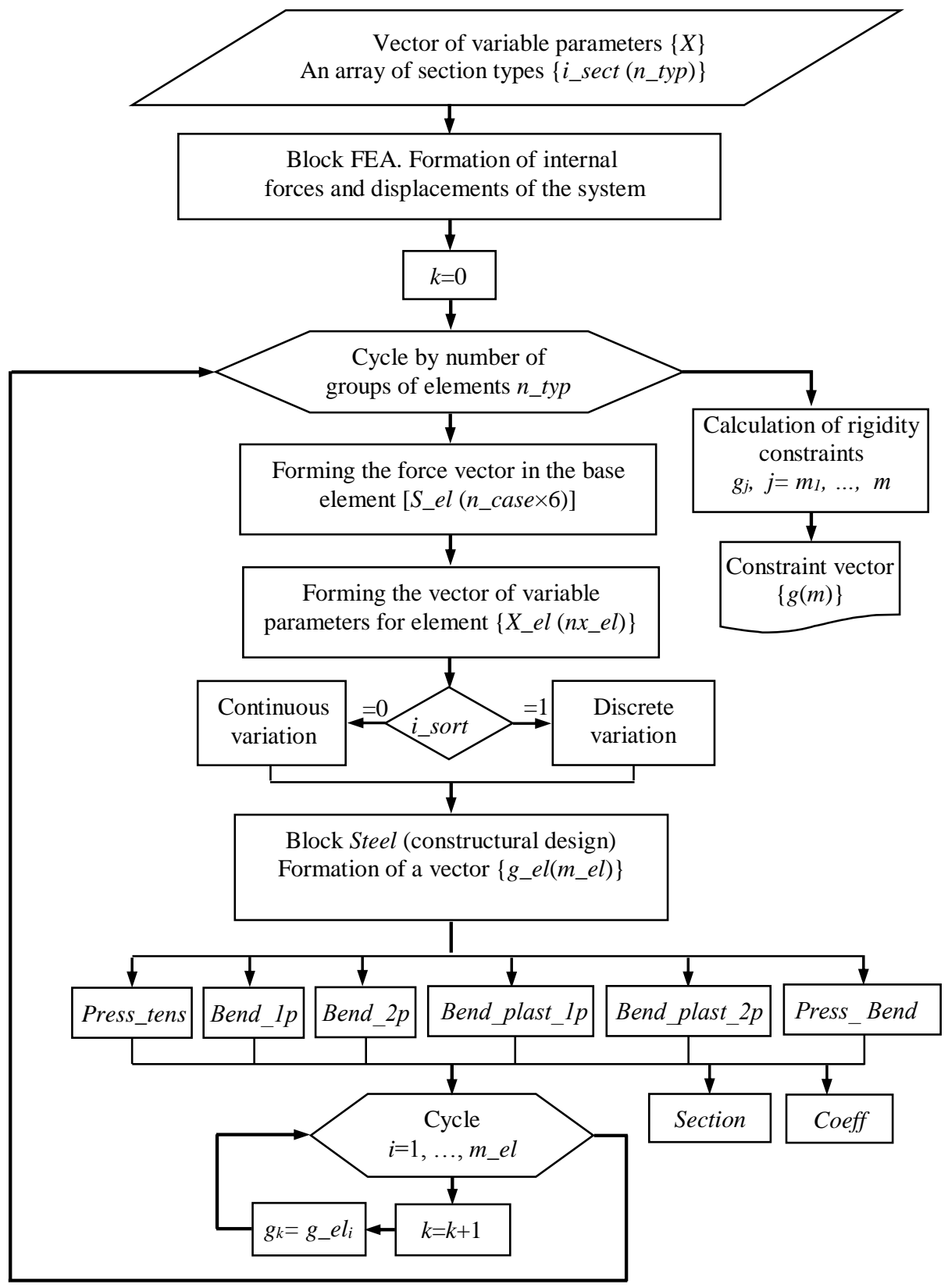

Fig. 4. Block diagram of the algorithm for the formation of constraint functions.

Constraint selection array is provided with the help of which any restrictions can be disabled. The constraint vector of the entire system, dimension $m$, contains contributions from individual groups (the number of groups is $n_{-} t y p$ ). This approach allows independently define the physical and geometric properties for each group of elements. The 
finite element analysis is performed to determine the internal force and displacements of the system. To access the Steel block where constructural design is implemented the corresponding tuning is made. A vector of variable parameters is formed for this group of elements - $\left\{X \_e l\left(n x \_e l\right)\right\}$. The mode of continuous variation $\left(i \_s o r t=0\right)$ or the mode of selection of sections by assortments $\left(i \_s o r t=1\right)$ is set. From the internal force vector of the entire system an array of the forces of the group [S_el], containing 6 components for each section in the element, taking into account the specified number of loads ( $n_{-}$case), is extracted. A base element that has an unfavorable combination of forces is selected forming an array $\left[S \_e l\right]$ from a group of elements.

Each module of normative calculation refers to the library Section where the geometric characteristics of the cross sections listed in Table 3 are calculated. The Coeff module realizes the calculation of all necessary normative coefficients.

There is another group of restrictions on the stiffness which are specified in the form of tolerances for the movement of nodes in the algorithm.

$$
g=\begin{array}{r}
\Delta_{k} \\
{[\Delta]}
\end{array}-1 \leq 0 .
$$

Fig. 5 shows the possible options for such nodal displacements.
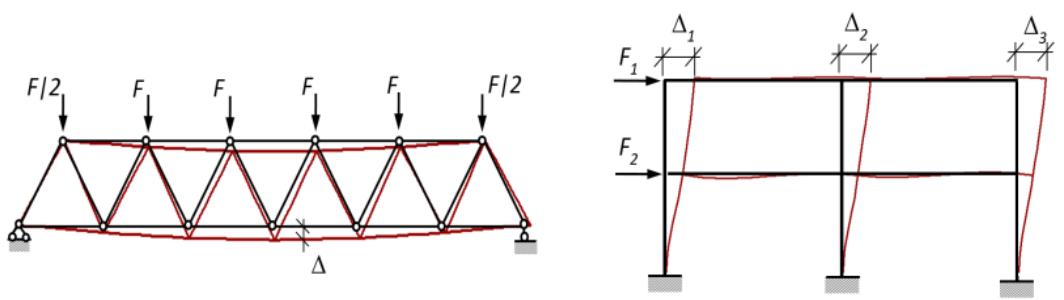

Fig. 5. Displacement the nodes of the calculation scheme.

\section{Example of optimizing the spatial carcass of a two-story building}

Based on the above algorithm the software complex ROSC (Calculation and optimization of steel structures) was developed. It allows solving practical problems [16]. We give an example of the optimal design of the frame of a two-story building, the design scheme of which is shown in Fig. 6.

The parameters of the cross sections as well as the angle of inclination of the roof vary here.

$$
\begin{aligned}
& \text { The volume of the carcass is } \quad f(x)=\sum_{i=1}^{34} A_{i}(x) \cdot l_{i}(x), \\
& \text { minimized: }
\end{aligned}
$$

where $A_{i}(x), l_{i}(x)$ is the cross-sectional area and the length of the $i$-th element.

The functions of restrictions are: a) regulatory checks on strength and stability; b) tolerances for the maximum displacement of nodes.

The problem is solved with on-going changes of cross-section parameters (variant 1) and with discrete change of parameters of sections according to the metal products standard (variant 2). 
a)

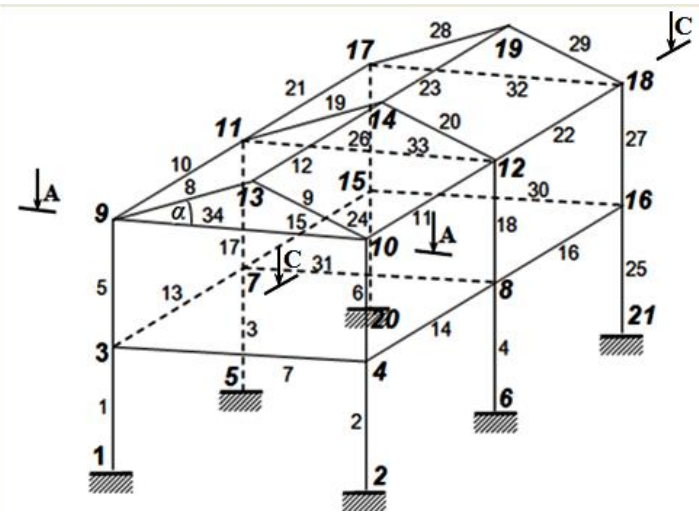

b)

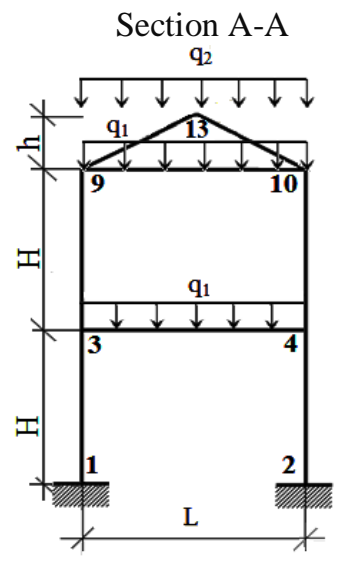

Section C-C

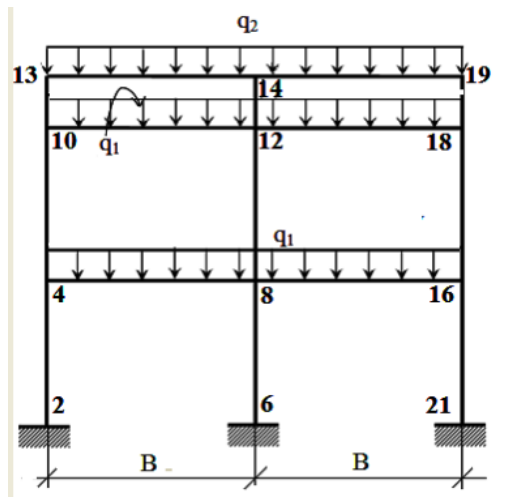

c)
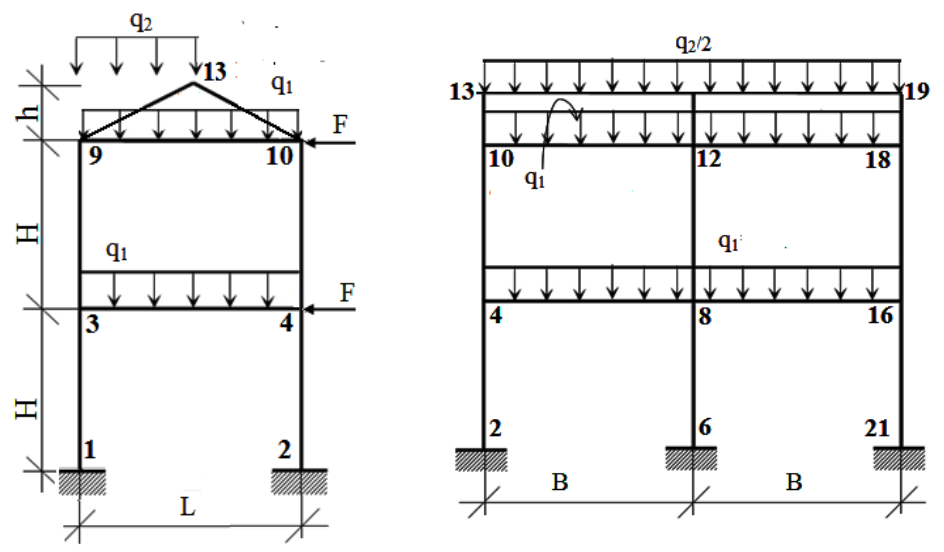

Fig. 6. a) The design scheme of the frame b) The first case of loading c) The second case of loading.

\section{Initial data:}

- frame dimensions $2 \mathrm{~L} \times \mathrm{B} \times \mathrm{H}(\mathrm{L}=6 \mathrm{~m}, \mathrm{~B}=6 \mathrm{~m}, \mathrm{H}=3,6 \mathrm{~m})$;

- distributed load: $\mathrm{q}_{1}=25,8 \mathrm{kN} / \mathrm{m} ; \mathrm{q}_{2}=7,18 \mathrm{kN} / \mathrm{m}$; 
- wind load $\mathrm{F}=10 \mathrm{kN}$;

- material: steel C345 $\left(\mathrm{Ry}=32 \mathrm{kN} / \mathrm{cm}^{2}\right)$;

- modulus of elasticity $\mathrm{E}=21000 \mathrm{kN} / \mathrm{cm}^{2}$;

- cross-section of columns;

- cross-section of horizontal and inclined elements: I

- number of loading cases $=2$;

- horizontal movement tolerance $[\Delta \max ]=1,8 \mathrm{~cm}$ (1/400 of the building height);

- number of groups of elements $=5$;

- the range of angle $\alpha$ is as follows: $\alpha_{\min }=5^{0}, \alpha_{\max }=45^{\circ}$;

- specified accuracy in constraints $=10^{-3}$.

The results of solving the optimization problem

Fig. 7 shows a graph of the change in the objective function at iterations for variant 1 when the parameters change continuously. It is shown that already at the first iteration there is a rapid convergence to a close solution to the optimum. The number of iterations can be significantly reduced if we specify a lower accuracy in the discrepancy constraints (for example within 5\%).

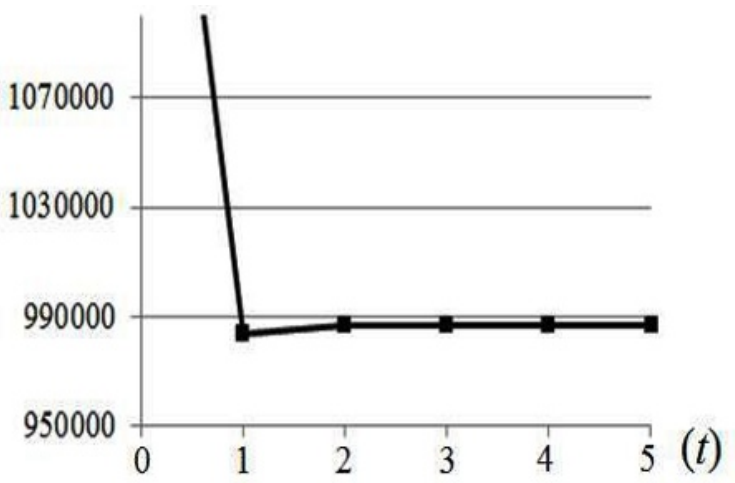

Fig. 7. Changing of the objective function on iterations $t$ (variant 1).

The resulting solution was investigated for uniqueness by specifying several initial projects. The spread of optimal values in the first variant was $0,0016 \%$. Three local solutions were obtained with a spread of the objective function of $5,7 \%$. In the second variant with a discrete variation of the parameters. The main results of the calculation are given in Table 4.

Table 4. Results of optimization of the spatial framework.

\begin{tabular}{|l|c|c|}
\hline Indicators & Variant 1 & Variant 2 \\
\hline Angle of inclination $\alpha$ (deg.) & $27^{0}$ & $18^{0}$ \\
\hline Number of iterations & 5 & 10 \\
\hline $\begin{array}{l}\text { Number of accesses to functions of } \\
\text { constraints }\end{array}$ & 170163 & 4896 \\
\hline Max. discrepancy in constraints & $-0,46 \cdot 10^{-3}$ & $-0,87 \cdot 10^{-2}$ \\
\hline Volume $\left(\mathrm{cm}^{3}\right)$ & 986987,702 & 1065521,59 \\
\hline Volume $(\%)$ & $79,69 \%$ & $100 \%$ \\
\hline
\end{tabular}

\section{Conclusion}


The proposed algorithm covers a wide range of optimization problems for rod structures according to the minimum material consumption criterion. The possibility of an optimal choice of cross-sections of structures as well as the external geometry of the structure (coordinates of the nodes of the design scheme) is considered.

A software package for calculating and optimizing steel structures has been developed on the basis of the algorithm. It makes possible to determine the geometric parameters of structures taking into account the regulatory requirements, the fulfillment of which is specified with a certain degree of accuracy. The stability of the algorithm is confirmed by calculations from various initial projects.

\section{References}

1. S. S. Rao, Engineering optimization. Theory and practice (John Wiley \& Sons, 2009)

2. D. Rees, Mechanics of Optimal Structural Design: Minimum Weight Structures (Wiley ed. 2009).

3. E. J. Haug, J. S. Arora, Applied Optimal Design: Mechanical And Structural Systems, (Wiley-Interscience, NY, 1979).

4. H. Adeli, K. Sarma, JNME, 55(12) (2002)

5. J. Farkas, Optimum Design of Metal Structures (NY: Horwood Halsted Press, 1984)

6. J. Farkas, K. Jarmai, Economic Design of Metal Structures (Millpress, 2003)

7. E. S Kameshki, M. P. Saka, JCSR, 59 (2003)

8. E. S Kameshki, M. P. Saka, C\&S, 1-2 (2007)

9. O. C. Zienkiewicz, The finite element method in engineering science (London; NY: McGraw-Hill, 1971)

10. K. J. Bathe, Finite Element Procedures. Prentice Hall, Englewood (New Jersey, 1996)

11. R. H. Gallagher, Finite element analysis: Fundamentals (Prentice Hall, Englewood Cliffs, N.J., 1975)

12. D. P. Berteskas, Nonlinear Programming (Athena Scientific, Belmont, MA, 1995)

13. M. Avriel, B. Golany, Mathematical Programming for Industrial Engineers (Marcel and Dekker, NY, 1996)

14. D. M. Himmelblau, Applied Nonlinear Programming, NY (1972)

15. T. L. Dmitrieva, Le Tran Minh Dat, IJCCSE, 12(4) (2016)

16. T. L. Dmitrieva, Le Tran Minh Dat, 10(2) (2012) 